\title{
Inflationary Cosmology
}

\author{
By MICHAEL S. T URNER $\mathbf{R}^{1,2} \dagger$ \\ ${ }^{1}$ Departments of Physics and of Astronomy and Astrophysics, Enrico Fermi Institute, The \\ University of Chicago, Chicago, IL 60637-1433, USA \\ ${ }^{2}$ NASA/Fermilab Astrophysics Center, Fermilab, Batavia, IL 60510-0500, USA
}

\begin{abstract}
Inflation is a bold and expansive extension of the Standard Cosmology. It holds the promise to extend our understanding of the Universe to within $10^{-32} \mathrm{sec}$ of the big bang and answer most of the pressing questions in cosmology. Its boldest assertion is that all the structure observed in the Universe today arose from quantum-mechanical fluctuations on subatomic scales. A flood of cosmological observations and laboratory experiments are testing inflation and within five years we should know whether inflation is to become part of a new standard cosmology.
\end{abstract}

\section{From Hubble to Zel'dovich \& Novikov}

The value of the Hubble constant has changed by about a factor of ten since Edwin Hubble's pioneering measurements. The context in which we view the Universe has changed even more profoundly. Until 1964 cosmology was mostly concerned with cosmography; the spirit of this period was perhaps best captured by Sandage, "the quest for two numbers $\left(H_{0}\right.$ and $\left.q_{0}\right)$." The discovery of the Cosmic Background Radiation (CBR) led to the establishment of a physical foundation for the expanding Universe - the hot big-bang cosmology. The 1970s saw this model become firmly established as the Standard Cosmology. Igor Novikov played an important role in this enterprise, and with Zel'dovich wrote one of the first reviews of the Standard Cosmology (Novikov \& Zel'dovich 1967) as well as a comprehensive and authoritative summary of it in Volume 2 of Relativistic Astrophysics (Structure and Evolution of the Universe) (Zel'dovich \& Novikov 1983).

The hot big-bang cosmology is a remarkable achievement. It provides a reliable account of the Universe from about $10^{-2}$ sec to the present (Peebles et al. 1991; Turner 1993a). Further, the hot big-bang model together with modern ideas in particle physics - the Standard Model, supersymmetry, grand unification, and superstring theory - provides a framework for sensible speculation all the way back to the Planck epoch, and perhaps even earlier. Here too, Novikov was one of the pioneering figures in the study of the earliest moments (Novikov \& Zel'dovich 1973; Zel'dovich \& Novikov 1983).

Building upon the Standard Cosmology, in the 1980s cosmologists and particle physicists began an ambitious program to root the hot big-bang model in fundamental physics and to address a deeper set of questions. What is the nature of the ubiquitous dark matter that is the dominant component of the mass density? Why does the Universe contain only matter? What is the origin of the tiny inhomogeneities that seeded the formation of structure, and how did that structure evolve? Why is the portion of the Universe that we can see so flat and smooth? What is the value of the cosmological constant? What is the origin of the entropy (photons in the CBR)? How did the expansion begin-or was there a beginning? What was the big bang?

In the past fifteen years much progress has been made, and many believe that the answers to all these questions involve events that took place during the earliest moments and involved physics beyond the Standard Model (Kolb \& Turner 1990). For example, the matter-antimatter asymmetry, quantified as a net baryon number of about $10^{-10}$

$\dagger$ This work was supported by the US DoE (at Chicago and Fermilab) and the US NASA (at Fermilab). 
per photon, is believed to have developed through interactions that do not conserve baryon number or $C, C P$ and occurred out of thermal equilibrium. If "baryogenesis" involved unification-scale physics the baryon asymmetry developed around $10^{-34} \mathrm{sec}$ (Kolb \& Turner 1983); on the other hand, baryogenesis may have occurred at the weak scale $\left(T \sim 300 \mathrm{GeV}\right.$ and $\left.t \sim 10^{-11} \mathrm{sec}\right)$ through baryon-number violation within the Standard Model and $C, C P$ violation from physics beyond the Standard Model (Cohen et al. 1992; Dolgov 1992).

The most optimistic early-Universe cosmologists (of which I am one) believe that we are on the verge of solving almost all of the above problems and extending our knowledge of the Universe back to around $10^{-32}$ sec after "the bang." The key is inflation. Among other things, inflation has led to the cold dark matter model of structure formation, whose basic tenets are scale-invariant density perturbations and dark matter whose primary composition is slowly moving elementary particles (e.g., axions or neutralinos). Cold dark matter provides a crucial test of inflation and a possible window to physics at unificationscale energies. If cold dark matter is correct, it would complete the Standard Cosmology by connecting the theorist's smooth early Universe to the astronomer's Universe which abounds with structure, galaxies, clusters of galaxies, superclusters, voids and great walls (Huchra \& Geller 1989).

\section{Inflation}

As successful as the big-bang cosmology is, it suffers from a dilemma involving initial data. Extrapolating back, one finds that the Universe apparently began from a very special state: A slightly inhomogeneous and very flat Robertson-Walker spacetime. Collins and Hawking showed that the set of initial data that evolve to a spacetime that is as smooth and flat as ours is today is of measure zero (Collins \& Hawking 1973). [In the context of simple grand unified theories, the hot big-bang model suffers from another serious problem: the extreme overproduction of superheavy magnetic monopoles (Preskill 1984); in fact, attempting to solve the monopole problem led Guth to inflation.]

The cosmological appeal of inflation is the lessening of the dependence of the present state of the Universe upon its initial state. Two elements are essential: (1) accelerated ("superluminal") expansion and the concomitant growth of the scale factor; and (2) massive entropy production ( $\mathrm{Hu}$, Turner \& Weinberg 1994). Through inflation, a small, smooth subhorizon-sized patch of the early Universe grows to a large enough size and comes to contain enough heat (entropy in excess of $10^{88}$ ) to encompass our present Hubble volume. In addition, superluminal expansion guarantees that the Universe today appears flat (just as any small portion of the surface of a large sphere appears flat).

While there is presently no standard model of inflation - just as there is no standard model for physics at these energies (typically $10^{15} \mathrm{GeV}$ or so) - viable models have much in common. They are based upon well posed, albeit highly speculative, microphysics involving the classical evolution of a scalar field. A nearly exponential expansion is driven by the potential energy ("vacuum energy") that arises when the scalar field is displaced from its potential-energy minimum. Provided the potential is flat, during the time it takes for the field to roll to the minimum of its potential the Universe undergoes many e-foldings of expansion (more than around 60 or so are required to realize the beneficial features of inflation). As the scalar field nears the minimum, the vacuum energy has been converted to coherent oscillations of the scalar field, which correspond to nonrelativistic scalar-field particles. The eventual decay of these particles into lighter particles and their thermalization results in the "reheating" of the Universe and accounts for all the heat in the Universe today (the entropy production event). 
The growth of the cosmic scale factor (by a factor greater than that since the end of inflation) allows quantum fluctuations excited on very small scales $\left(\lesssim 10^{-23} \mathrm{~cm}\right)$ to be stretched to astrophysical scales $\left(\gtrsim 10^{25} \mathrm{~cm}\right)$. Quantum fluctuations in the scalar field responsible for inflation ultimately lead to an almost scale-invariant spectrum of density perturbations (Guth \& Pi 1982; Hawking 1982; Starobinsky 1982; Bardin, Steinhardt \& Turner 1983), and quantum fluctuations in the metric itself lead to an almost scaleinvariant spectrum of gravity-waves (Rubakov, Sazhin \& Veryaskin 1984; Fabbri \& Pollock 1983; Starobinsky 1983a Abbott \& Wise 1984). Scale invariance for density perturbations means scale-independent fluctuations in the gravitational potential (equivalently, density perturbations of different wavelength cross the horizon with the same amplitude); scale invariance for gravity waves means that gravity waves of all wavelengths cross the horizon with the same amplitude. Because of subsequent evolution, neither the scalar nor the tensor perturbations are scale invariant today.

\subsection{Grander Implications}

Inflation lessens greatly the "specialness" problem, but does not eliminate it (Turner \& Widrow 1986; Jensen \& Schabes 1987; Starobinsky 1983b). All open FRW models will inflate and become flat; however, many closed FRW models will recollapse before they can inflate. If one imagines the most general initial spacetime as being comprised of negatively and positively curved FRW (or Bianchi) models that are stitched together, the failure of the positively curved regions to inflate is of little consequence: because of exponential expansion during inflation the negatively curved regions will occupy most of the space today. Inflation does not solve the smoothness problem forever; it just postpones the problem into the exponentially distant future: We will be able to see outside our smooth inflationary patch at a time $t \sim t_{0} \exp \left[3\left(N-N_{\min }\right]\right.$, where $N$ is the actual number of e-foldings of inflation and $N_{\min } \sim 60$ is the minimum required to solve the horizon/flatness problems.

Linde has emphasized that inflation has changed our view of the Universe in a very fundamental way (Linde 1990). While cosmologists have long used the Copernician principle to argue that the Universe must be smooth because of the smoothness of our Hubble volume, in the post-inflation view, our Hubble volume is smooth because it is a small part of a region that underwent inflation. On the largest scales the structure of the Universe is likely to be very rich: Different regions may have undergone different amounts of inflation, may have different realizations of the laws of physics because they evolved into different vacuum states (of equivalent energy), and may even have different numbers of spatial dimensions. Since it is likely that most of the volume of the Universe is still undergoing inflation and that inflationary patches are being constantly produced (eternal inflation), the age of the Universe is a meaningless concept and our expansion age merely measures the time back to our big bang - the nucleation of our inflationary bubble.

\subsection{Specifics}

Guth's seminal paper (Guth 1981) both introduced the idea of inflation and showed that the model that he based the idea upon did not work! Thanks to very important contributions by Linde (Linde 1982) and Albrecht and Steinhardt (Albrecht \& Steinhardt 1982) that was quickly remedied, and today there are many viable models of inflation. That of course is good news and bad news - since it means that there is no standard model of inflation. The absence of a standard model of inflation should of course be viewed in the light of our general ignorance about physics at unification-scale energies.

Many different approaches have taken in constructing particle-physics models for in-

flation. Some have focussed on very simple scalar potentials, e.g., $V(\phi)=\lambda \phi^{4}$ or 
$=m^{2} \phi^{2} / 2$, without regard to connecting the model to any underlying theory (Linde 1983 ; Steinhardt \& Turner 1984). Others have proposed more complicated models that attempt to make contact with speculations about physics at very high energies, e.g., grand unification (Shafi \& Vilenkin 1984, Pi 1984) supersymmetry (Holman, Ramond \& Ross 1984; Olive 1990; Murayama 1994: Randall, Soljacic \& Guth 1996) preonic physics (Cvetic et al. 1990), or supergravity (Copeland et al. 1994). Several authors have attempted to link inflation with superstring theory (Gasperini \& Veneziano 1994: Brustein \& Veneziano 1994; Banks et al. 1995) or "generic predictions" of superstring theory such as pseudo-Nambu-Goldstone boson fields (Freese, Frieman, \& and Olinto 1990). While the scale of the vacuum energy that drives inflation is typically of order $\left(10^{15} \mathrm{GeV}\right)^{4}$, a model of inflation at the electroweak scale, vacuum energy $\approx(1 \mathrm{TeV})^{4}$, has been proposed (Knox \& Turner 1993). There are also models in which there are multiple epochs of inflation (Silk \& Turner 1986; ;ofman, Linde \& Einsato 1987).

In all of the models above gravity is described by general relativity. A qualitatively different approach is to consider inflation in the context of alternative theories of gravity. The most successful of these models is first-order inflation (La \& Steinhardt 1991; Kolb 1991). First-order inflation returns to Guth's original idea of a strongly first-order phase transition; in the context of general relativity Guth's model failed because the phase transition, if inflationary, never completed. In theories where the effective strength of gravity evolves, like Brans-Dicke theory, the weakening of gravity during inflation allows the transition to complete. In other models based upon nonstandard gravitation theory, the scalar field responsible for inflation is itself related to the size of additional spatial dimensions, and inflation then also explains why our three spatial dimensions are so big, while the other spatial dimensions are so small.

All models of inflation have one feature in common: the scalar field responsible for inflation has a very flat potential-energy curve and is very weakly coupled. Invariably, this leads to a very small dimensionless number, usually a coupling constant of the order of $10^{-14}$. Such a small number, like other small numbers in physics (e.g., the ratio of the weak to Planck scales $\approx 10^{-17}$ or the ratio of the mass of the electron to the $W / Z$ boson masses $\approx 10^{-5}$ ), runs counter to one's belief that a truly fundamental theory should have no tiny parameters, and cries out for an explanation. At the very least, this small number must be stabilized against quantum corrections - which it is in all of the previously mentioned models. potential is related to other small numbers in particle physics: for example, the ratio of the electron mass to the weak scale or the ratio of the unification scale to the Planck scale. Explaining the origin of the small number that seems to be associated with inflation is both a challenge and an opportunity.

Because of the growing base of observations that bear on inflation, another approach to model building is emerging: the use of observations to constrain the underlying inflationary model. In Section 4 I will discuss the possibilities for reconstructing the inflationary potential.

$\dagger$ After all, inflation probably involves physics at energy scales not too different from the Planck scale and the effective theory of gravity at these energies could well be very different from general relativity; in fact, there are some indications from superstring theory that gravity in these circumstances might be described by a Brans-Dicke-like theory.

$\ddagger$ It is sometimes stated that inflation is unnatural because of the small coupling of the scalar field responsible for inflation; while the small coupling certainly begs explanation, these inflationary models are not unnatural in the technical sense as the small number is stable against quantum fluctuations. 


\subsection{Three Robust Predictions}

While there are many varieties of inflation, there are three robust predictions which are crucial to sharply testing inflation. (9

(a) Flat universe. Because solving the "horizon" problem (large-scale smoothness in spite of small particle horizons at early times) and solving the "flatness" problem (maintaining $\Omega$ very close to unity until the present epoch) are linked geometrically (Kolb \& Turner 1990; Hu, Turner \& Weinberg 1994), this is the most robust prediction of inflation. Said another way, it is the prediction that most inflationists would be least willing to give up. [Even so, models of inflation have been constructed where the amount of inflation is tuned just to give $\Omega_{0}$ less than one today (Bucher, Goldhaber \& Turok 1995; Bucher \& Turok 1995).] Through the Friedmann equation for the scale factor, flatness implies that the total energy density (matter, radiation, vacuum energy, and anything else) is equal to the critical density.

(b) Nearly scale-invariant spectrum of gaussian density perturbations. Essentially all inflation models predict a nearly scale-invariant spectrum of gaussian density perturbations. Described in terms of a power spectrum, $P(k) \equiv\left\langle\left|\delta_{k}\right|^{2}\right\rangle=A k^{n}$, where $\delta_{k}$ is the Fourier transform of the primeval density perturbations, and the spectral index $n=1$ in the scale-invariant limit. The overall amplitude $A$ is model dependent. Density perturbations give rise to CBR anisotropy as well as seeding structure formation. Requiring that the density perturbations are consistent with the observed level of anisotropy of the CBR (and large enough to produce the observed structure formation) is the most severe constraint on inflationary models and leads to the small dimensionless number that all inflationary models have.

(c) Nearly scale-invariant spectrum of gravitational waves. These gravitational waves have wavelengths from around $1 \mathrm{~km}$ to the size of the present Hubble radius and beyond. Described in terms of a power spectrum for the dimensionless gravity-wave amplitude at early times, $P_{T}(k) \equiv\left\langle\left|h_{k}\right|^{2}\right\rangle=A_{T} k^{n_{T}-3}$, where the spectral index $n_{T}=0$ in the scale-invariant limit. Once again, the overall amplitude $A_{T}$ is model dependent (varying as the value of the inflationary vacuum energy). Unlike density perturbations, which are required to initiate structure formation, there is no cosmological lower bound to the amplitude of the gravity-wave perturbations. Tensor perturbations also give rise to CBR anisotropy; requiring that they do not lead to excessive anisotropy implies that the energy density that drove inflation must be less than about $\left(10^{16} \mathrm{GeV}\right)^{4}$. This indicates that if inflation took place, it did so at an energy well below the Planck scale.fi

There are other interesting consequences of inflation that are less generic. For example, in first-order inflation, where reheating occurs through the nucleation and collision of vacuum bubbles, there is an additional, larger amplitude, but narrow-band, spectrum of gravitational waves $\left(\Omega_{\mathrm{GW}} h^{2} \sim 10^{-6}\right.$ ) (Turner \& Wilczek 1990; Kosowsky, Turner \& Watkins 1992). In other models large-scale primeval magnetic fields of interesting size are seeded during inflation (Turner \& Widrow 1988; Ratra 1992).

I want to emphasize the importance of the tensor perturbations. The attractiveness of a flat Universe with scale-invariant density perturbations was appreciated long before inflation. Verifying these two predictions of inflation, while important, will not provide a "smoking gun." A spectrum of nearly scale-invariant tensor perturbations is a defining

I Because theorists are so clever, it is not possible nor prudent to use the word immutable. Models that violate any or all of these "robust predications" can and have been constructed.

$\dagger$ To be more precise, the part of inflation that led to perturbations on scales within the present horizon involved subPlanckian energy densities. In some models of inflation, the earliest stage of inflation, which only influences scales much larger than the present horizon, involve energies as large as the Planck energy density. 
feature of inflation, and further, is crucial to obtaining information about the underlying scalar potential.

CBR anisotropy probably provides the best possibility of detecting the tensor perturbations, but their contribution to CBR anisotropy has to be separated that of the scalar perturbations. Because the sky is finite, sampling variance sets a fundamental limit: the tensor contribution to CBR anisotropy can only be separated from that of the scalar if $T / S$ is greater than about 0.14 (Knox, L. \& Turner 1994) ( $T$ is the contribution of tensor perturbations to the variance of the CBR quadrupole and $S$ is the same for scalar perturbations).

It is possible that the stochastic background of gravitational waves itself can be directly detected, though it appears that the LIGO facilities being built will lack the sensitivity and even space-based interferometry (e.g., LISA) is not a sure bet (Turner, Lidsey \& White 1993; Turner 1996).

\section{Cold Dark Matter}

Cold dark matter actually draws from three important ideas - inflation, big-bang nucleosynthesis, and the quest to better understand the fundamental forces and particles. As discussed above, inflation predicts a flat Universe (total energy density equal to the critical density) and nearly scale-invariant density perturbations. Big-bang nucleosynthesis provides the most precise determination of the density of ordinary matter, present density between $1.5 \times 10^{-31} \mathrm{~g} \mathrm{~cm}^{-3}$ and $4.5 \times 10^{-31} \mathrm{~g} \mathrm{~cm}^{-3}$, or fraction of critical density $\Omega_{B}=$ $0.008 h^{-2}-0.024 h^{-2}$, where $H_{0}=100 h \mathrm{~km} \mathrm{~s}^{-1} \mathrm{Mpc}^{-1}$ (Copi, Schramm \& Turner 1995). Allowing $h=0.4-0.8$, consistent with modern measurements (Fukugita, Hogan \& Peebles 1993; Jacoby G. et al. 1992), implies that ordinary matter can contribute at most $10 \%$ of the critical density. If the inflationary prediction is correct, then most of the matter in the Universe must be nonbaryonic.

This idea has received indirect support from particle physics. Attempts to further our understanding of the particles and forces have led to the prediction of new, stable or long-lived particles that interact very feebly with ordinary matter. These particles, if they exist, should have been present in great numbers during the earliest moments and remain today in numbers sufficient to contribute the critical density (Turner 1991). Two of the most attractive possibilities behave like cold dark matter: a neutralino of mass $10 \mathrm{GeV}$ to $1000 \mathrm{GeV}$, predicted in supersymmetric theories, and an axion of mass $10^{-6} \mathrm{eV}$ to $10^{-4} \mathrm{eV}$, needed to solve a subtle problem of the standard model of particle physics (strong-CP problem). The third interesting possibility is that one of the three neutrino species has a mass between $5 \mathrm{eV}$ and $30 \mathrm{eV}$; neutrinos move very fast and are referred to as hot dark matter. H

According to cold dark matter theory CDM particles provide the cosmic infrastructure: It is their gravitational attraction that forms and holds cosmic structures together. Structure forms in a hierarchical manner, with galaxies forming first and successively larger objects forming thereafter (Blumenthal et al. 1984). Quasars and other rare objects form at redshifts of up to five, with ordinary galaxies forming a short time later. Today, superclusters, objects made of several clusters of galaxies, are just becoming bound by the gravity of their CDM constituents. The formation of larger and larger

$\dagger$ The possibility that most of the exotic particles are fast-moving neutrinos - hot dark matter - was explored first and found to be inconsistent with observations (White, Frenk \& Davis 1983). The problem is that large structures form first and must fragment into smaller structures, which conflicts with the fact that large structures are just forming today. 
objects continues. In the clustering process regions of space are left devoid of matter and galaxies - leading to voids.

\subsection{Standard Cold Dark Matter}

When the cold dark matter scenario emerged more than a decade ago many referred to it as a no parameter theory because it was so specific compared to previous models for the formation of structure. This was an overstatement as there are cosmological quantities that must be known to determine the development of structure in detail. However, the data available did not require precise knowledge of these quantities to test the model.

Broadly speaking these parameters can be organized into two groups. First are the cosmological parameters: the Hubble constant, specified by $h$; the density of ordinary matter, specified by $\Omega_{B} h^{2}$; the power-law index $n$ and normalization $A$ that quantify the density perturbations; and the amplitude and spectral index $n_{T}$ that quantify the gravitational waves. The inflationary parameters fall into this category because there is no standard model of inflation. On the other hand, once determined they can be used to discriminate between models of inflation.

The other quantities specify the composition of invisible matter in the Universe: radiation, dark matter, and a possible cosmological constant. Radiation refers to relativistic particles: the photons in the CBR, three massless neutrino species (assuming none of the neutrino species has a mass), and possibly other undetected relativistic particles (some particle-physics theories predict the existence of additional massless particle species). At present relativistic particles contribute almost nothing to the energy density in the Universe, $\Omega_{R} \simeq 4.2 \times 10^{-5} h^{-2}$; early on - when the Universe was smaller than about $10^{-5}$ of its present size - they dominated the energy content.

In addition to CDM particles, the dark matter could include other particle relics. For example, each neutrino species has a number density of $113 \mathrm{~cm}^{-3}$, and a neutrino species of mass $5 \mathrm{eV}$ would account for about $20 \%$ of the critical density $\left(\Omega_{\nu}=\right.$ $\left.m_{\nu} / 90 h^{2} \mathrm{eV}\right)$. Predictions for neutrino masses range from $10^{-12} \mathrm{eV}$ to several $\mathrm{MeV}$, and there is some experimental evidence that at least one of the neutrino species has a small mass (Parke 1995; Athanassopoulos et al. 1995; Hill 1995; Hirata et al. 1992; Fukuda et al. 1994; Becker-Szendy 1992).

Finally, there is the cosmological constant. Both introduced and abandoned by Einstein, it is still with us. In the modern context it corresponds to an energy density associated with the quantum vacuum. At present, there is no reliable calculation of the value that the cosmological constant should take, and so its existence must be regarded as a logical possibility (Weinberg 1989).

The original no parameter cold dark matter model, referred to as standard CDM, is characterized by: $h=0.5, \Omega_{B}=0.05, \Omega_{\mathrm{CDM}}=0.95, n=1$, no gravitational waves and standard radiation content. The overall normalization of the density perturbations was fixed by comparing the predicted level of inhomogeneity with that seen today in the distribution of bright galaxies. Specifically, the amplitude $A$ was determined by comparing the expected mass fluctuations in spheres of radius $8 h^{-1} \mathrm{Mpc}$ (denoted by $\sigma_{8}$ ) to the galaxy-number fluctuations in spheres of the same size. The galaxy-number fluctuations on the scale $8 h^{-1} \mathrm{Mpc}$ are unity; adjusting $A$ to achieve $\sigma_{8}=1$ corresponds to the assumption that light, in the form of bright galaxies, traces mass. Choosing $\sigma_{8}$ to be less than one means that light is more clustered than mass and is a biased tracer of mass. There is some evidence that bright galaxies are somewhat more clumped than mass with biasing factor $b \equiv 1 / \sigma_{8} \simeq 1-2$ (Lin 1996).

A dramatic change occurred with the detection of CBR anisotropy by COBE in 1992 (Smoot et al. 1992). The COBE measurement permitted a precise normalization of the 


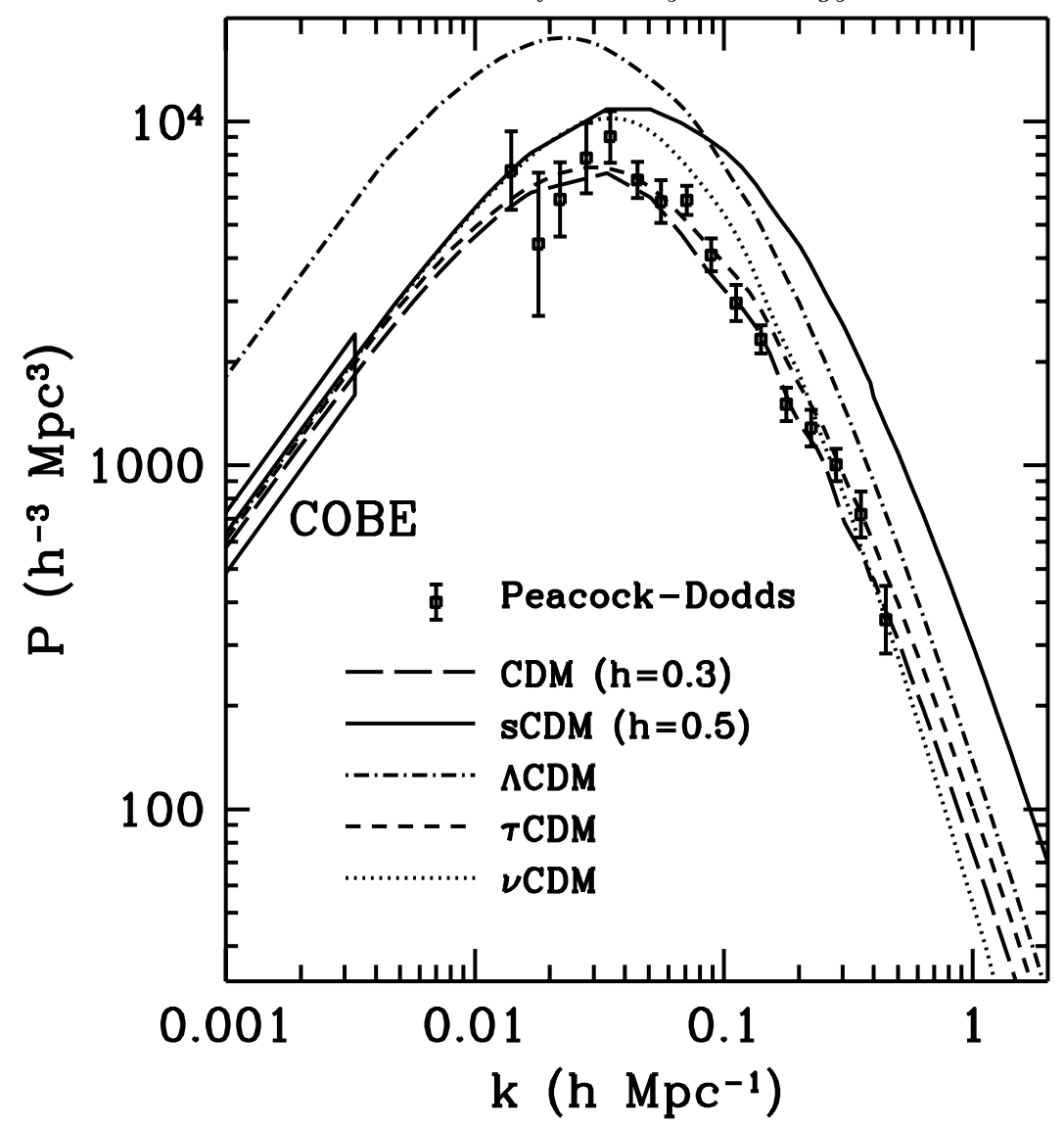

Figure 1. Measurements of the power spectrum, $P(k) \equiv\left|\delta_{k}\right|^{2}$, and the predictions of different COBE-normalized CDM models. The points are from several redshift surveys as analyzed in Ref. (Peacock \& Dodds 1994); the models are: $\Lambda$ CDM with $\Omega_{\Lambda}=0.6$ and $h=0.65$; standard CDM (sCDM), CDM with $h=0.35 ; \tau \mathrm{CDM}$ (with the energy equivalent of 12 massless neutrino species) and $\nu$ CDM with $\Omega_{\nu}=0.2$ (unspecified parameters have their standard CDM values).

amplitude of density perturbations on very large scales $\left(\lambda \sim 10^{4} h^{-1} \mathrm{Mpc}\right)$ without regard to the issue of biasing. [CBR anisotropy on the angular scale $\theta$ arises primarily due to inhomogeneity on length scales $\lambda \sim 100 h^{-1} \mathrm{Mpc}(\theta / \mathrm{deg})$.] For standard CDM, the COBE normalization leads to: $\sigma_{8}=1.2 \pm 0.1$ or anti-bias since $b=1 / \sigma_{8} \simeq 0.7$. The pre-COBE normalization $\left(\sigma_{8}=0.5\right)$ led to too little power on scales of $30 h^{-1} \mathrm{Mpc}$ to $300 h^{-1} \mathrm{Mpc}$, as compared to what was indicated in redshift surveys, the angular correlations of galaxies on the sky and the peculiar velocities of galaxies. The COBE normalization leads to about the right amount of power on these scales, but predicts too much power on small scales $\left(\lesssim 8 h^{-1} \mathrm{Mpc}\right)$; see Fig. 1 .

While standard CDM is in general agreement with the observations, a consensus has developed that the conflict just mentioned is probably significant (Ostriker 1993; Liddle \& Lyth 1993). This has led to a new look at the cosmological and invisiblematter parameters and to the realization that the problems of standard CDM are simply a poor choice for the standard parameters. 


\section{A Flood of Data}

Standard CDM has served well to focus everyone's attention on the same problem. However, the quality and quantity of data have improved and knowledge of the cosmological and invisible-matter parameters has become important for serious testing of CDM and inflation. There are a variety of combinations of the parameters that lead to good agreement with the existing data on both large and small length scales - and thus can make a claim to being the new standard CDM model. Figure 2 shows the allowed values of the cosmological parameters for several COBE-normalized CDM models.f.

More precisely, for a given CDM model - specified by the cosmological and invisiblematter parameters - the expected CBR anisotropy is computed and required to be consistent with the four-year COBE data set at the two-sigma level (Bennett et al. 1996; Gorski et al. 1996: White \& Bunn 1995). The expected level of inhomogeneity in the Universe today is compared to three robust measurements of inhomogeneity: the shape of the power spectrum as inferred from surveys of the distribution of galaxies today (Peacock \& Dodds 1994); a determination of $\sigma_{8}$ based upon the abundance of rich, xray emitting clusters (White, Efstathiou \& Frenk 1993); and the abundance of hydrogen clouds at high redshift (which probes early structure formation) (Lanzetta, Wolfe \& Turnshek 1995; Storrie-Lombardi et al. 1995).

Figure 2 summarizes the overall picture. The simplest CDM models - those with standard invisible-matter content - lie in a region that runs diagonally from smaller Hubble constant and larger $n$ to larger Hubble constant and smaller $n$. That is, higher values of the Hubble constant require more tilt (tilt referring to deviation from scale invariance). Note too that standard CDM is well outside of the allowed range. Current measurements of CBR anisotropy on the degree scale, as well as the COBE fouryear anisotropy data, preclude $n$ less than about 0.7 (see Fig. 3). This implies that the largest Hubble constant consistent with the simplest CDM models is slightly less than $60 \mathrm{~km} \mathrm{~s}^{-1} \mathrm{Mpc}^{-1}$. If the invisible-matter content is nonstandard, higher values of the Hubble constant can be accommodated. In Fig. $2, \Omega_{\nu}$ is taken to be 0.2 ; in fact, this is essentially the largest value allowed by measurements of the power spectrum (Dodelson, Gates \& Turner 1996; Ma \& Bertschinger 1994; Liddle et al. 1995). On the other hand, even $\Omega_{\nu}=0.05$ (around $1 \mathrm{eV}$ worth of neutrinos) can have important consequences (e.g., accommodating a higher value of the Hubble constant or more nearly scale-invariant density perturbations).

Changes in the different parameters from their standard CDM values alleviate the excess power on small scales in different ways. Tilt has the effect of reducing power on small scales when power on very large scales is fixed by COBE. A small admixture of hot dark matter works because fast moving neutrinos suppress the growth of inhomogeneity on small scales by streaming from regions of higher density and to regions of lower density. (It was in fact this feature of hot dark matter that led to the demise of the hot dark matter model for structure formation.)

A low value of the Hubble constant, additional radiation or a cosmological constant all reduce power on small scales by lowering the ratio of matter to radiation. Since the critical density depends upon the square of the Hubble constant, $\rho_{\text {Critical }}=3 H_{0}^{2} / 8 \pi G$, a smaller value corresponds to a lower matter density since $\rho_{\text {Matter }}=\rho_{\text {Critical }}$ for a

$\dagger$ Computation of both the CBR anisotropy and the level of inhomogeneity today depends upon the invisible-matter content and the cosmological parameters and requires that the distribution of matter and radiation be evolved numerically; for details see Refs. (Peebles \& Yu 1970; Wilson \& Silk 1981: Bond \& Efstathiou, 1984; Holtzman 1989; van Dalen \& Schaefer 1992; Sugiyama \& Gouda 1992; Dodelson \& Jubas 1993). The discussion of viable models is a summary of collaborative work (Dodelson, Gates \& Turner 1996). 


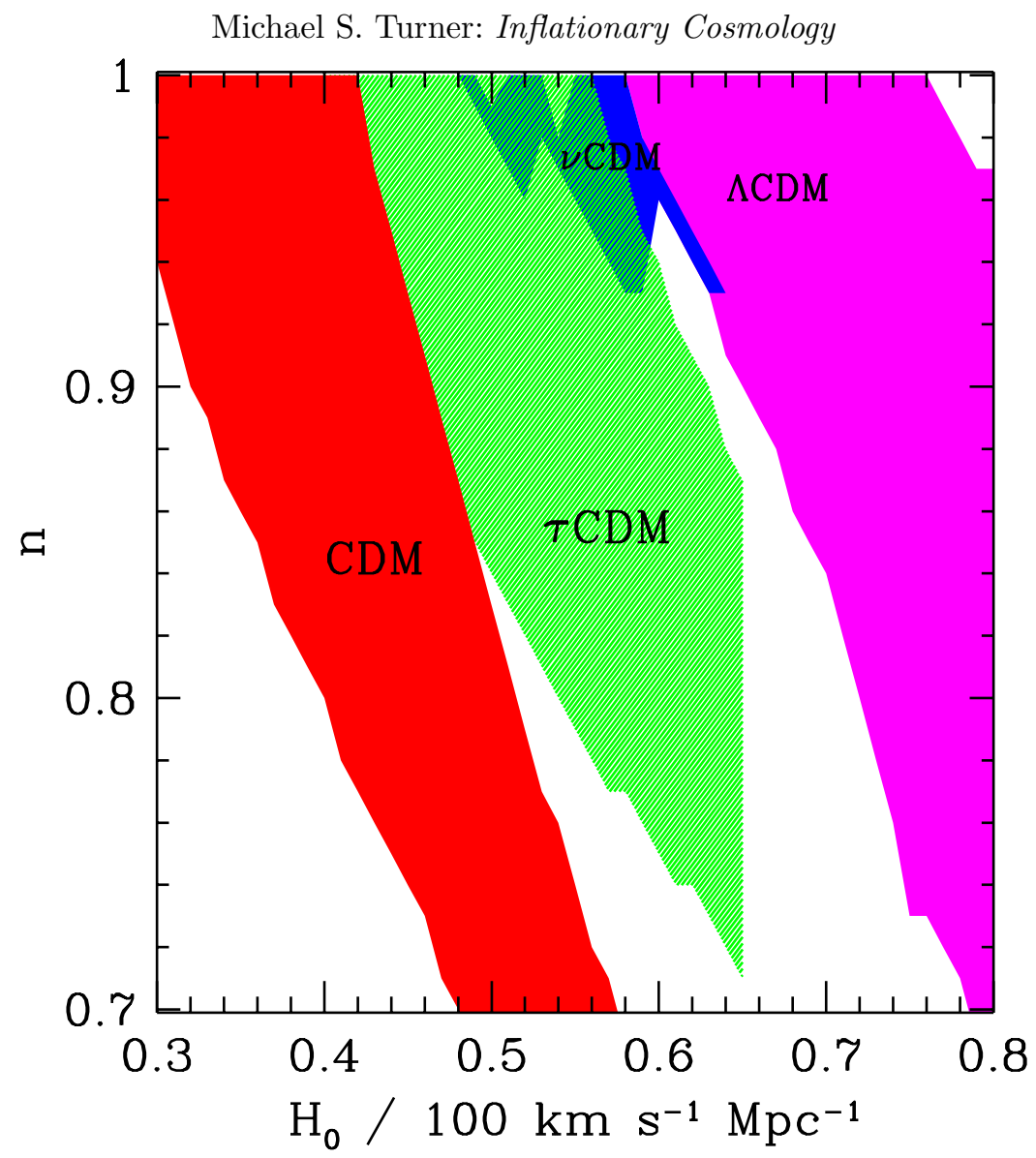

Figure 2. Acceptable values of the cosmological parameters $n$ and $h$ for CDM models with standard invisible-matter content (CDM), with $20 \%$ hot dark matter $(\nu \mathrm{CDM})$, with additional relativistic particles (the energy equivalent of 12 massless neutrino species, denoted $\tau \mathrm{CDM}$ ), and with a cosmological constant that accounts for $60 \%$ of the critical density $(\Lambda \mathrm{CDM})$. Note that standard CDM $(n=1$ and $h=0.5)$ is not viable.

flat Universe without a cosmological constant. Shifting some of the critical density to vacuum energy also reduces the matter density since $\Omega_{\text {Matter }}=1-\Omega_{\Lambda}$. Lowering the ratio of matter to radiation reduces the power on small scales in a subtle way. While the primeval fluctuations in the gravitational potential are nearly scale-invariant, density perturbations today are not because the Universe made a transition from an early radiation-dominated phase ( $t \lesssim 1000 \mathrm{yrs}$ ), where the growth of density perturbations is inhibited, to the matter-dominated phase, where growth proceeds unimpeded. This introduces a feature in the power spectrum today (see Fig. 1), whose location depends upon the relative amounts of matter and radiation. Lowering the ratio of matter to radiation shifts the feature to larger scales and with power on large scales fixed by COBE this leads to less power on small scales.

Some of the viable models have been discussed as singular solutions - cosmological constant (Turner, Steigman \& Krauss 1984, Turner 1991; Peebles 1984; Efstathiou et al. 1990; Kofman \& Starobinsky 1985), very low Hubble constant (Bartlett et al. 1995), tilt (Cen et al. 1992) Davis et al. 1992; Lucchin, Mattarese \& Mollerach 1992: Salopek 1992; Liddle \& Lyth 1992; Lidsey \& Coles 1992;; Souradeep \& Sahni 1992; |Adams et al. 1993), tilt + low Hubble constant (White et al. 1995), extra radiation (Dodelson, Gyuk \& 


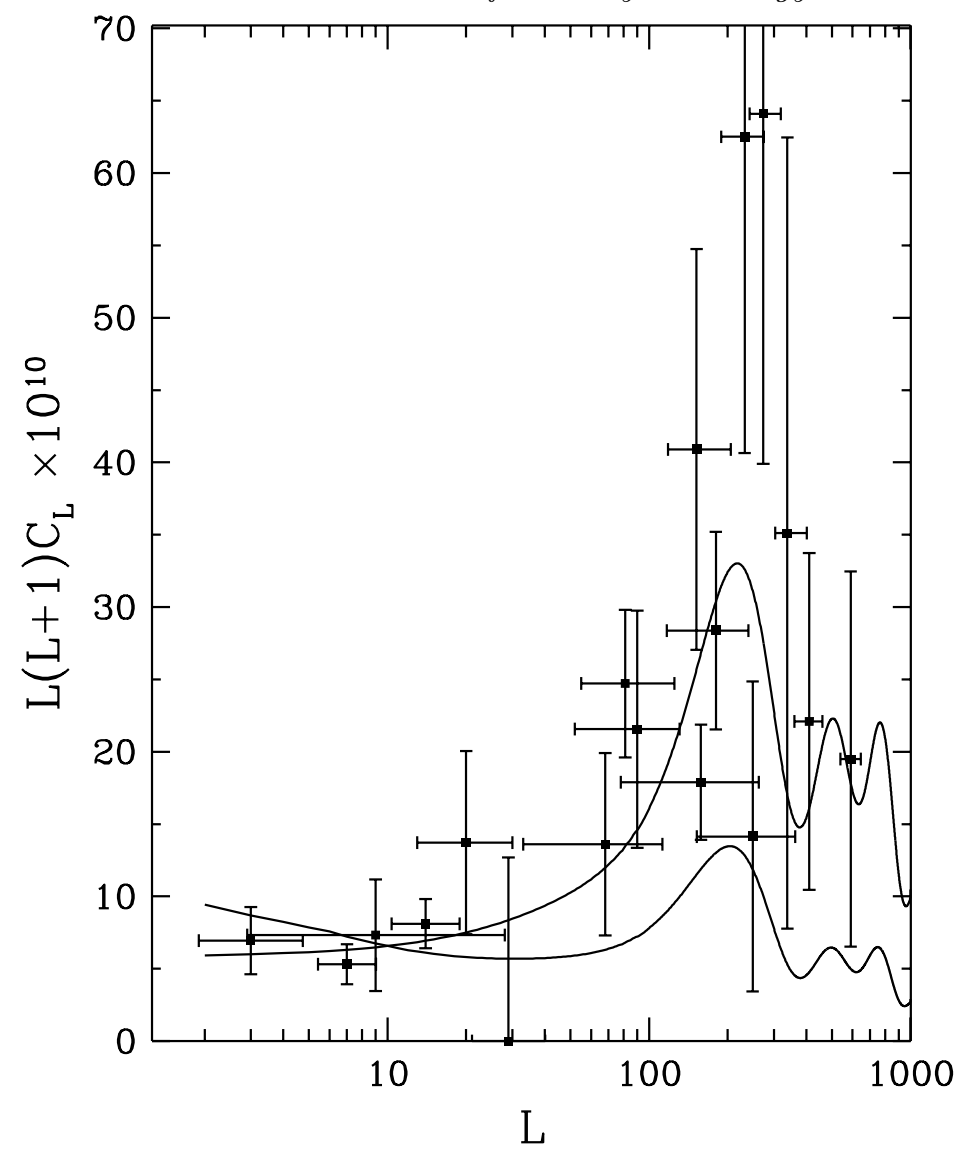

FiguRE 3. Summary of CBR anisotropy measurements and predictions for three CDM models [adapted from Ref. (White, Scott, \& and Silk 1995)]. Plotted are the squares of the measured multipole amplitudes $\left(C_{l}=\left\langle\left|a_{l m}\right|^{2}\right\rangle\right)$ in units of $7 \times 10^{-10}$ vs. multipole number $l$. The temperature difference on angular scale $\theta$ is given roughly by $\sqrt{l(l+1) C_{l}}$ with $l \sim 200^{\circ} / \theta$. The theoretical curves are standard CDM and CDM with $n=0.7$ and $h=0.5$.

Turner 1994; Bond \& Efstathiou 1991), an admixture of hot dark matter (Shafi \& Stecker 1984; Davis, Summers \& Schlegel 1992; Primack et al. 1995; Pogosyan \& Starobinsky 1995). There is actually a continuum of viable models, as can be seen in Fig. 2, which arises because of imprecise knowledge of cosmological parameters and the invisible-matter sector and not the inventiveness of theorists.

\subsection{Other and Future Considerations}

There are many other observations that bear on structure formation. However, with cosmological data systematic error and interpretational issues are important considerations. In fact, if all extant observations were taken at face value, there is no viable model for structure formation, cold dark matter or otherwise! With this as a preface, I now discuss some of the other existing data as well as future measurements that will more sharply test cold dark matter.

There is tension between measures of the age of the Universe and determinations of the Hubble constant. It arises because determinations of the ages of the oldest stars lie between 13 Gyr and 19 Gyr (Chaboyer et al. 1996, Bolte \& Hogan 1995) and recent measurements of the Hubble constant favor values between $60 \mathrm{~km} \mathrm{~s}^{-1} \mathrm{Mpc}^{-1}$ and 


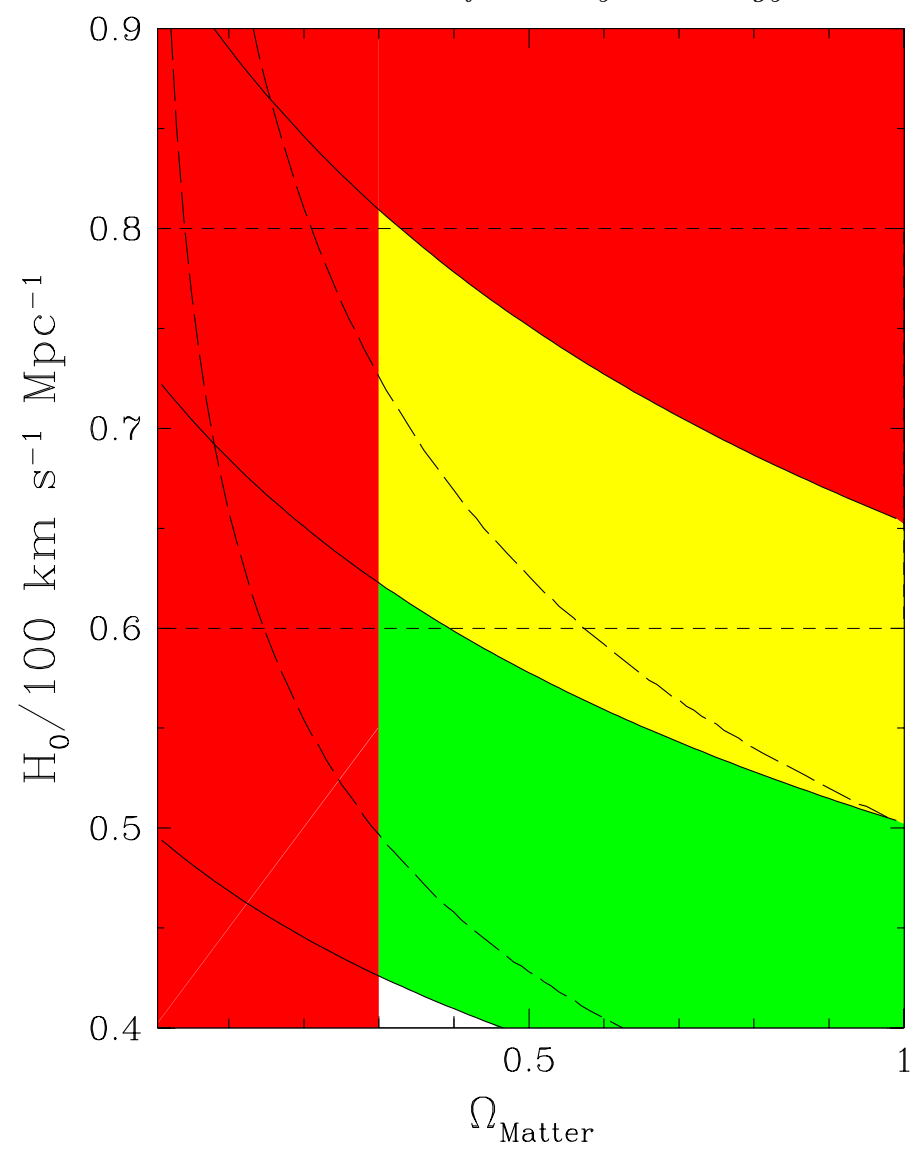

FiguRE 4. Isochrones in the $H_{0}-\Omega_{\text {Matter }}$ plane. The bottom band corresponds to time back to the bang of between $13 \mathrm{Gyr}$ and $19 \mathrm{Gyr}$; the lightest band to between $10 \mathrm{Gyr}$ and $13 \mathrm{Gyr}$; the darkest region is disallowed: $\Omega_{\text {Matter }}<0.3$ or expansion time less than $10 \mathrm{Gyr}$. Broken horizontal lines indicate the range favored for the Hubble constant, $80 \mathrm{~km} \mathrm{~s}^{-1} \mathrm{Mpc}^{-1}>H_{0}>60 \mathrm{~km} \mathrm{~s}^{-1} \mathrm{Mpc}^{-1}$. The age - Hubble constant tension is clear, especially for the inflationary prediction of $\Omega_{\text {Matter }}=1$. The broken curves denote the $13 \mathrm{Gyr}-19 \mathrm{Gyr}$ isochrone for $\Lambda \mathrm{CDM}$; a cosmological constant greatly lessens the tension.

$80 \mathrm{~km} \mathrm{~s}^{-1} \mathrm{Mpc}^{-1}$ Reiss, Kirshner \& Press 1995; Hamuy 1995. Freedman et al. 1994), which, for $\Omega_{\text {Matter }}=1$, leads to a time back to the bang of 11 Gyr or less (see Fig. 4).fi These age determinations receive additional support from estimates of the age of the galaxy based upon the decay of long-lived radioactive isotopes and the cooling of whitedwarf stars, and all methods taken together make a strong case for an absolute minimum age of 10 Gyr (Cowan, Thieleman \& Truran 1991). It should be noted that within the uncertainties there is no inconsistency, even for $\Omega_{\text {Matter }}=1$.

While age is not a major issue for cold dark matter - large-scale structure favors an older Universe by virtue of a lower Hubble constant or cosmological constant (see Fig. 5) - the Hubble constant still has great leverage. If it is determined to be greater than about $60 \mathrm{~km} \mathrm{~s}^{-1} \mathrm{Mpc}^{-1}$, then only CDM models with nonstandard invisible-matter content - a cosmological constant or additional radiation - can be consistent with large-

$\dagger$ The time back to the bang depends upon $H_{0}, \Omega_{\text {Matter }}$ and $\Omega_{\Lambda}$; for $\Omega_{\text {Matter }}=1$ and $\Omega_{\Lambda}=0$, $t_{\mathrm{BB}}=\frac{2}{3} H_{0}^{-1}$, or $13 \mathrm{Gyr}$ for $h=0.5$ and $10 \mathrm{Gyr}$ for $h=0.65$. For a flat Universe with a cosmological constant the numerical factor is larger than $2 / 3$ (see Fig. 4). 


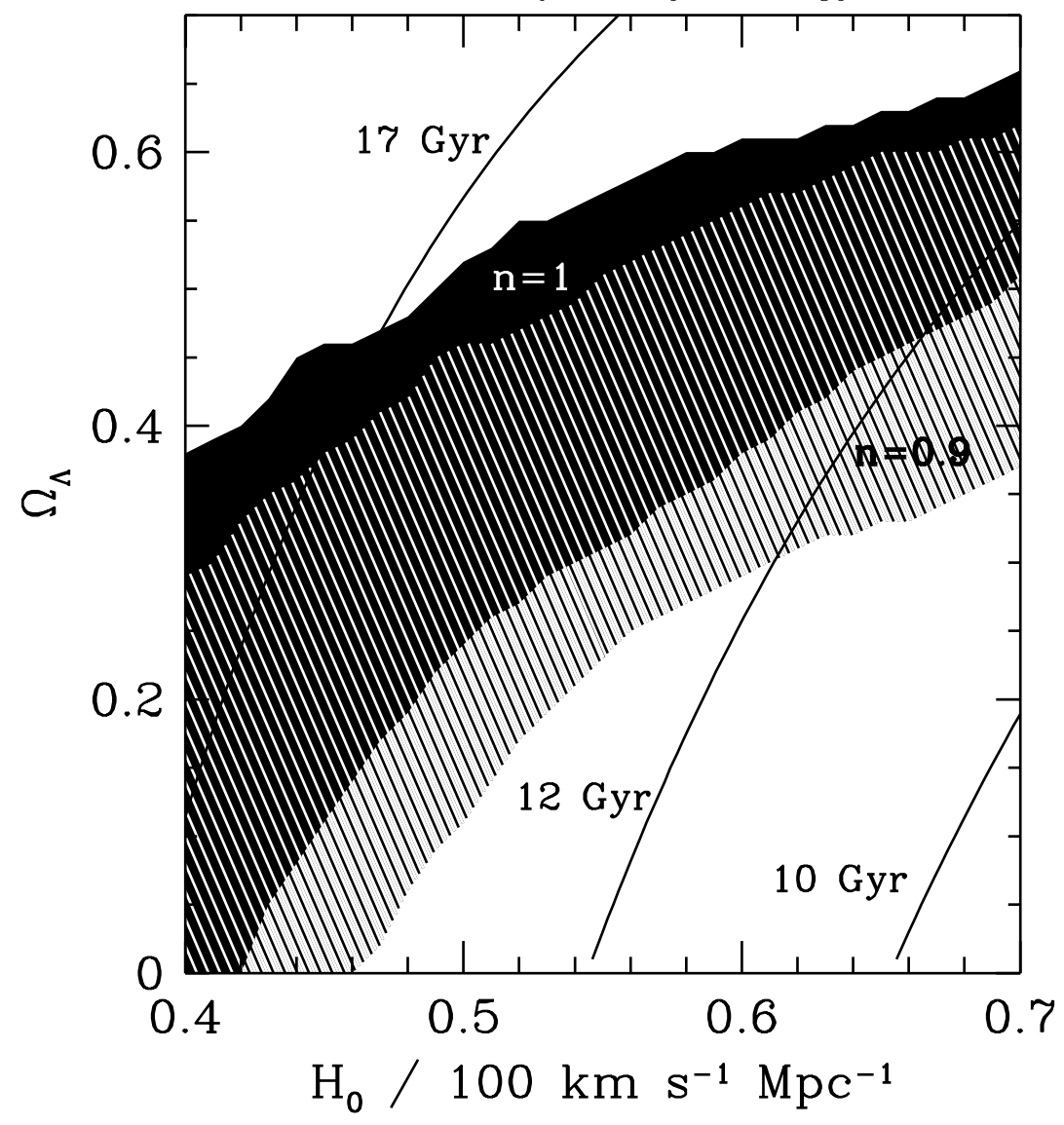

Figure 5. Acceptable values of $\Omega_{\Lambda}$ and $h$ for $n=0.9,1.0$. Note that large-scale structure considerations generally favor a more aged Universe - smaller $h$ or larger $\Omega_{\Lambda}$.

scale structure. If $H_{0}$ is greater than $65 \mathrm{~km} \mathrm{~s}^{-1} \mathrm{Mpc}^{-1}$, consideration of the age of the Universe leaves $\Lambda \mathrm{CDM}$ as the lone possibility. The issue of $H_{0}$ is not settled, but the use of Type Ia supernovae as standard candles, the study of Cepheid variable stars in nearby galaxies using the Hubble Space Telescope, and other methods make it likely that it will be soon.

If CDM is correct, baryons make up a small fraction of matter in the Universe. Most of the baryons in galaxy clusters are in the hot, x-ray emitting intracluster gas and not the luminous galaxies. The measured x-ray flux fixes the mass in baryons, while the measured x-ray temperature fixes the total mass (through the virial theorem). The baryon-tototal-mass has been determined from x-ray measurements for more than ten clusters and is found to be $M_{\mathrm{B}} / M_{\mathrm{TOT}} \simeq(0.04-0.1) h^{-3 / 2}$ (White et al. 1993; Briel et al. 1992; White \& Fabian 1995). Because of their size, clusters should represent a fair sample of the cosmos and thus the baryon-to-total mass ratio should reflect its universal value, $\Omega_{B} / \Omega_{\text {Matter }} \simeq(0.01-0.02) h^{-2} / \Omega_{\text {Matter }}$. These two ratios are consistent for models with a very low Hubble constant, $h \sim 0.4$ and $\Omega_{\text {Matter }}=1$, or with a cosmological constant and $\Omega_{\text {Matter }} \sim 0.3$. However, important assumptions are made in this analysis - that the hot gas is unclumped and in virial equilibrium and that magnetic fields do not provide significant pressure support for the gas - if any one of them is not valid the actual baryon 
fraction would be smaller (Babul \& Katz 1993), allowing for consistency with a larger value of $H_{0}$ without recourse to a cosmological constant.

The halos of individual spiral galaxies like our own are not large enough to provide a fair sample of matter in the Universe - for example, much of the baryonic matter has undergone dissipation and condensed into the disk of the galaxy. Nonetheless, the content of halos is expected to be primarily CDM particles. This is consistent with the fact that visible stars, hot gas, dust, and even dark stars acting as microlenses (known as MACHOs) account for only a fraction of the mass of our own halo Alcock et al. 1995; Gates, Gyuk \& Turner 1995; Bahcall et al. 1994; Flynn, Gould \& Bahcall 1996).

Determining the mean mass density of the Universe would discriminate between models with and without a cosmological constant, as well as test the inflationary prediction of a flat Universe. A definitive determination is still lacking. The measurement that averages over the largest volume - and thus is potentially most useful - uses the peculiar velocities of galaxies. Peculiar velocities arise due to the inhomogeneous distribution of matter, and the mean matter density can be determined by relating the peculiar velocities to the observed distribution of galaxies. The results of this technique indicate that $\Omega_{\text {Matter }}$ is at least 0.3 and perhaps as large as unity (Strauss \& Willick 1995, Dekel 1994). Though not definitive, this provides strong evidence for the existence of nonbaryonic dark matter, a key aspect of cold dark matter.

A different approach to the mean density is through the deceleration parameter $q_{0}$, which quantifies the slowing of the expansion due to the gravitational attraction of matter in the Universe. Its value is given by $q_{0}=\frac{1}{2} \Omega_{\text {Matter }}-\Omega_{\Lambda}$ (vacuum energy actually leads to accelerated expansion) and can be determined by relating the distances and redshifts of distant objects. In all but the $\Lambda \mathrm{CDM}$ scenario, $q_{0}=0.5$; for $\Lambda \mathrm{CDM}, q_{0} \sim-0.5$. Two groups are trying to measure $q_{0}$ by using high redshift $(z \sim 0.4-0.7)$ Type Ia supernovae as standard candles; the preliminary results of one group suggest that $q_{0}$ is positive (Perlmutter et al. 1996). More than a dozen distant Type Ia supernovae were discovered this year and both groups should soon have enough to measure $q_{0}$ with a precision of \pm 0.2 .

Gravitational lensing of distant QSOs by intervening galaxies is another way to measure $q_{0}$, and the frequency of QSO lensing suggests that $q_{0}>-0.6$ (Kochanek 1996). The distance to a QSO of given redshift is larger for smaller $q_{0}$, and thus the probability for its being lensed by an intervening galaxy is greater.

The $10 \mathrm{~m}$ Keck Telescope and the Hubble Space Telescope are providing the deepest images of the Universe ever and are revealing details of galaxy formation as well as the formation and evolution of clusters of galaxies (Fukugita, Hogan \& Peebles 1996). The Keck has made the first detection of deuterium in high redshift hydrogen clouds (Songaila et al. 1994; Carswell et al. 1994: Rugers \& Hogan 1996a; Tytler, Fan \& Burles 1996; Burles \& Tytler 1996" Wampler et al. 1996; Carswell, R. F. et al. 1996; Rugers \& Hogan 1996b; (Cowie \& Songaila 1996). This is a new confirmation of big-bang nucleosynthesis and has the potential of pinning down the density of ordinary matter to a precision of $10 \%$.

The level of inhomogeneity in the Universe today is determined largely from redshift surveys, the largest of which contain of order $10^{4}$ galaxies. Two larger surveys are in progress. The Sloan Digital Sky Survey will obtain positions for two-hundred million galaxies and redshifts for one million over a quarter of the sky; the Anglo - Australian Two-Degree Field will gather 250,000 redshifts in hundreds of two-degree fields. They will allow the power spectrum to be measured more precisely and out to large enough scales $\left(500 h^{-1} \mathrm{Mpc}\right)$ to connect with measurements from CBR anisotropy on angular scales of up to five degrees. 
The most fundamental element of cold dark matter - the existence of the CDM particles themselves - is being tested. While the interaction of CDM particles with ordinary matter occurs through very feeble forces and makes their existence difficult to test, experiments with sufficient sensitivity to detect the CDM particles that hold our own galaxy together if they are in the form of axions of mass $10^{-6} \mathrm{eV}-10^{-4} \mathrm{eV}$ (Rosenberg 1995) or neutralinos of mass tens of $\mathrm{GeV}$ (Caldwell 1995) are now underway. Evidence for the existence of the neutralino could also come from particle accelerators searching for other supersymmetric particles. In addition, several experiments sensitive to neutrino masses are operating or are planned, ranging from accelerator-based neutrino oscillation experiments to the detection of solar neutrinos to the study of the tau neutrino at $e^{+} e^{-}$colliders.

CBR anisotropy probes the power spectrum most cleanly as it is related directly to the distribution of matter when density perturbations were very small (Hu \& Sugiyama 1995). Current measurements are beginning to test CDM and differentiate between the variants (see Fig. 3); e.g., a spectral index $n<0.7$ is strongly disfavored. More than ten groups are making measurements with instruments in space, on balloons and at the South Pole. Two new satellite-borne experiments have been approved: NASA will launch MAP in 2001 and ESA will launch COBRAS/SAMBA in 2004. They will map the anisotropy of the CBR with better than $0.2^{\circ}$ resolution (more than 30 times better than COBE). The results from these maps should easily discriminate between the different variants of CDM (see Fig. 6).

The first and most powerful test to emerge from these measurements will be the location of the first (Doppler) peak in the angular power spectrum (see Fig. 6) (Kamionkowski, Spergel \& Sugiyama 1994; Jungman et al. 1996a; Hu \& White 1996). All variants of CDM predict the location of the first peak to lie in roughly the same place. On the other hand, in an open Universe (total energy density less than critical) the first peak occurs at a larger value of $l$ (much smaller angular scale). This will provide an important test of inflation. In addition, theoretical studies (Knox 1995; Jungman et al. 1996b) indicate that $n$ could be determined to a precision of a few percent, $\Omega_{\Lambda}$ to ten percent, and perhaps even $\Omega_{\nu}$ to enough precision to test $\nu$ CDM (Dodelson, Gates \& Stebbins 1996).

If all the current observations - from recent Hubble constant determinations to the cluster baryon fraction - are taken at face value, the cosmological constant + cold dark matter model is probably the best fit (Krauss \& Turner 1995: Ostriker \& Steinhardt 1995), though there may be a conflict with the measurement of $q_{0}$ with Type Ia supernovae. $\Lambda \mathrm{CDM}$ raises a fundamental question - the origin of the implied vacuum energy, about $\left(10^{-2} \mathrm{eV}\right)^{4}$ - since there is no known principle or mechanism that explains why it is less than $(300 \mathrm{GeV})^{4}$, let alone $\left(10^{-2} \mathrm{eV}\right)^{4}$ (Weinberg 1989). In any case, it would be imprudent to take all the observational data at face value because of important systematic and interpretational uncertainties. To paraphrase the biologist Francis Crick, a theory that fits all the data at any given time is probably wrong as some of the data are probably not correct.

\subsection{Reconstruction}

If inflation and the cold dark matter theory are shown to be correct, a window to the very early Universe $\left(t \sim 10^{-32} \mathrm{sec}\right)$ will have been opened. While it is certainly premature to jump to this conclusion, I would like to illustrate one example of what one could hope to learn. The spectra and amplitudes of the the tensor and scalar metric perturbations predicted by inflation depend upon the underlying model, to be specific, the shape of the inflationary scalar-field potential. If one can measure the power-law index of the scalar spectrum and the amplitudes of the scalar and tensor spectra, one can recover the value of the potential and its first two derivatives around the point on the potential where 


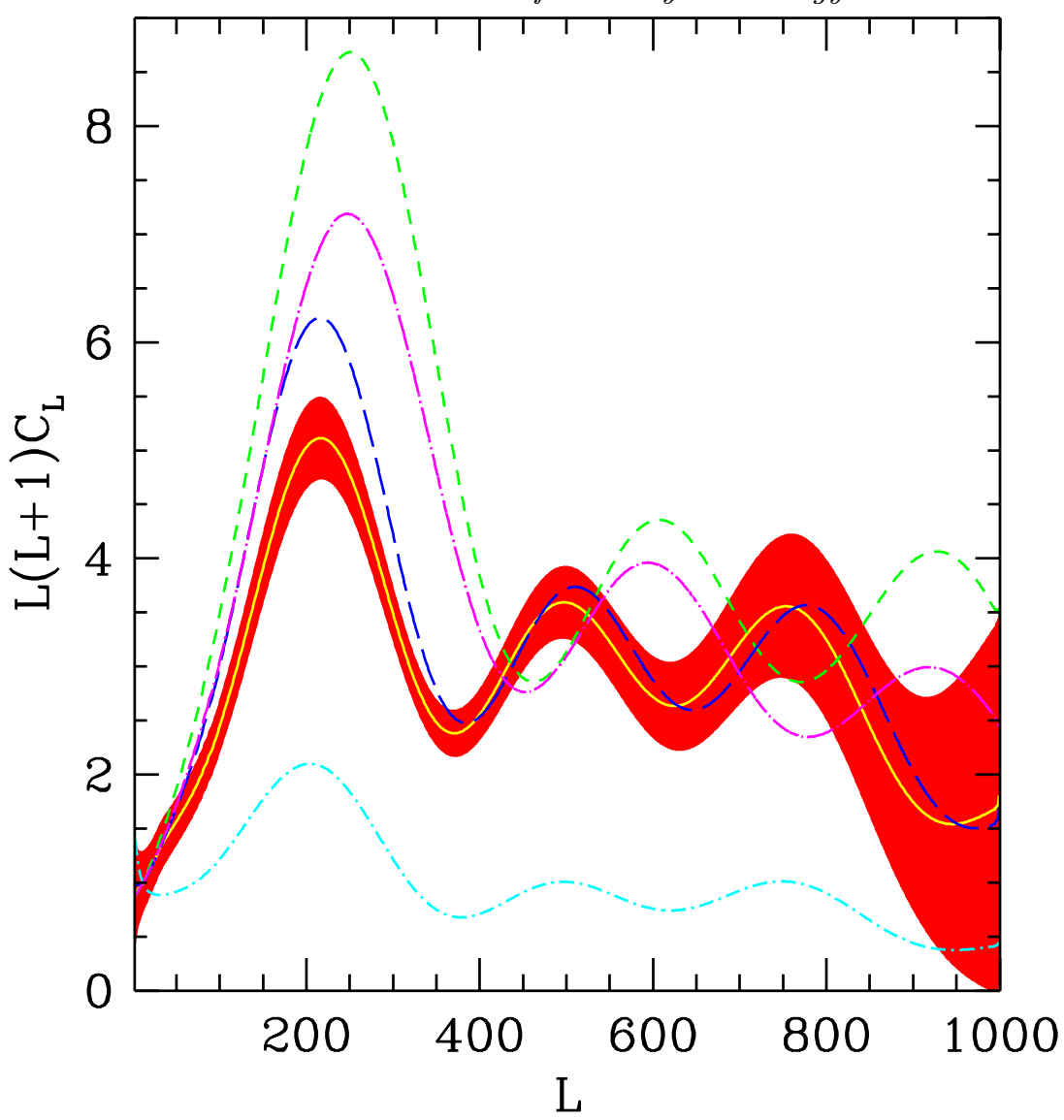

FiguRE 6. Predicted angular power spectra of CBR anisotropy for several viable CDM models and the anticipated uncertainty from a CBR satellite experiment with angular resolution of $0.3^{\circ}$. From top to bottom the models are: CDM with $h=0.35, \tau \mathrm{CDM}$ with the energy equivalent of 12 massless neutrino species, $\Lambda \mathrm{CDM}$ with $h=0.65$ and $\Omega_{\Lambda}=0.6, \nu \mathrm{CDM}$ with $\Omega_{\nu}=0.2$, and CDM with $n=0.7$ (unspecified parameters have their standard CDM values).

inflation took place (Turner 1993b, Lidsey et al. 1995):

$$
\begin{aligned}
V & =1.65 T m_{\mathrm{Pl}}^{4} \\
V^{\prime} & = \pm \sqrt{\frac{8 \pi r}{7}} V / m_{\mathrm{Pl}}, \\
V^{\prime \prime} & =4 \pi\left[(n-1)+\frac{3}{7} r\right] V / m_{\mathrm{Pl}}{ }^{2},
\end{aligned}
$$

where $r \equiv T / S$ ( $T$ is the contribution of tensor perturbations to the variance of the CBR quadrupole and $S$ is the same for scalar perturbations), prime indicates derivative with respect to $\phi, m_{\mathrm{Pl}}=1.22 \times 10^{19} \mathrm{GeV}$ is the Planck energy, and the sign of $V^{\prime}$ is indeterminate. In addition, if the tensor spectral index $n_{T}$ can be measured a consistency relation, $n_{T}=-r / 7$, can be used to test inflation. Reconstruction of the inflationary scalar potential would shed light on the underlying physics of inflation as well as physics at energies of the order of $10^{15} \mathrm{GeV}$. 


\section{Concluding Remarks}

Thanks to the work of Igor Novikov and others, the fifteen years following the discovery of the CBR saw the establishment of a physical basis for the expanding Universe, the hot big-bang cosmology. With this as a firm foundation, the decade of the 1980s has produced many bold and interesting speculations about the earliest history of the Universe which address the most fundamental problems in cosmology. Two of these ideas, inflation and cold dark matter, are so attractive that experimenters and observers took them seriously. The decade of the 1990s is producing a flood of data that are testing inflation and cold dark matter. The stakes for both cosmology and fundamental physics are high: if correct, inflation and cold dark matter represent a major extension of the big bang and our understanding of the Universe, which would certainly shed light on fundamental

physics at energies beyond the reach of terrestrial accelerators.

\section{REFERENCES}

Abbott, L. \& Wise, M. 1984 Nucl. Phys. B 244, 541.

Adams, F. C. et AL. 1993 Phys. Rev. D47, 426.

Albrecht, A. \& Steinhardt, P. J. 1982 Phys. Rev. Lett. 48, 1220.

AlCOCK, C. ET AL. 1995 Phys. Rev. Lett. 74, 2867.

Athanassopoulos, C. Et Al. 1995 Phys. Rev. Lett. 75, 2650.

BABul, A. \& Katz, N. 1993 Astrophys. J. 406, L51.

BAHCALL, J. ET AL. 1994 Astrophys. J. Lett. 435, L51.

BANKS, T. ET AL. 1995 hep-th/9503114.

Bardeen, J. M., Steinhardt, P. J. \& Turner, M. S. 1983 Phys. Rev. D 28, 697.

Bartlett, J. Et AL. 1995 Science 267, 980.

Becker-Szendy, R. et AL. 1992 Phys. Rev. D 46, 3720.

Bennett, C. L. et AL. 1996 Astrophys. J. (Lett.) in press, astro-ph/9601067.

Blumenthal, G. ET AL. 1984 Nature 311, 517.

Bolte, M. \& Hogan, C. J. 1995 Nature 376, 399.

Bond, J. R. \& Efstathiou, G. 1984 Astrophys. J. 285, L45.

Bond, J. R. \& Efstathiou, G. 1991 Phys. Lett. B 265, 245.

Briel, U. G. ET AL. 1992 Astron. Astrophys. 259, L31.

Brustein, R. \& Veneziano, G. 1994 Phys. Lett. B 329, 429.

Bucher, M., Goldhaber, A. S. \& Turok, N. 1995 Phys. Rev. D 52, 3314.

Bucher, M. \& Turok, N. 1995 hep-ph/9503393.

Burles, S. \& Tytler, D. 1996 astro-ph/9603070.

Caldwell, D. O. 1995 in Particle and Nuclear Astrophysics in the Next Millennium (eds. E.W. Kolb and R.D. Peccei), p. 38. World Scientific, Singapore.

Carswell, R. F. et Al. 1994 Mon. Not. Roy. Astr. Soc. 268, L1.

Carswell, R. F. et Al. 1996 Mon. Not. Roy. Astron. Soc. 278, 506.

Cen, R., Gnedin, N., Kofman, L., \& Ostriker, J. P. 1992 Astrophys. J. 399, L11.

Chaboyer, B., Demarque, P., Kernan, P. J. \& Krauss, L. M. 1996 Science 271, 957.

Cohen, A., Kaplan, D. \& Nelson, A. 1992 Annu. Rev. Nucl. Part. Sci. 43, 27.

Collins, C. B. \& Hawking, S. W. 1973 Astrophys. J. 180, 317.

Copeland, E. J. et Al. 1994 Phys. Rev. D 49, 6410.

Copi, C., Schramm, D. N. \& Turner, M. S. 1995 Science 267, 192.

Cowan, J., Thieleman, F. \& Truran, J. 1991 Ann. Rev. Astron. Astrophys. 29, 447. 
Cowie, L. \& Songaila, A. 1996 in preparation.

Cvetic, M., Hubsch, T., Pati, J. \& Stremnitzer, H. 1990 Phys. Rev. D 40, 1311.

Dalen, A. van \& Schaefer, R. K. 1992 Astrophys. J. 398, 33.

Davis, R. ET AL. 1992 Phys. Rev. Lett. 69, 1856.

Davis, M., Summers, F. \& Schlegel, D. 1992 Nature 359.

Dekel, A. 1994 Ann. Rev. Astron. Astrophys. 32, 319.

Dodelson, S., Gates, E. \& Stebbins, A. S. 1996 Astrophys. J., in press, astro-ph/9509147.

Dodelson, S., Gates, E. I. \& Turner, M. S. 1996 astro-ph/9603081.

Dodelson, S., Gyuk, G. \& Turner, M. S. 1994 Phys. Rev. Lett. 72, 3578.

Dodelson, S. \& Jubas, J. M. 1993 Phys. Rev. Lett. 70, 2224.

Dolgov, A. 1992 Phys. Repts. 222, 309.

Efstathiou, G. ET Al. 1990 Nature 348, 705.

FabBri, R. \& Pollock, M. 1983 Phys. Lett. B 125, 445.

Flynn, C., Gould, A. \& Bahcall, J. 1996 astro-ph/9603035.

Freedman, W. ET AL. 1994 Nature 371, 757.

Freese, K., Frieman, J. A. \& And Olinto, A. 1990 Phys. Rev. Lett. 65, 3233.

Fukuda, Y. ET AL. 1994 Phys. Lett. B 335, 237.

Fukugita, M., Hogan, C. J. \& Peebles, P. J. E. 1993 Nature 366, 309.

Fukugita, M., Hogan, C. J. \& Peebles, P. J. E. 1996 Nature 381, 489.

Gasperini, M. \& Veneziano, G. 1994 Phys. Rev. D 50, 2519.

Gates, E., Gyuk, G. \& Turner, M. S. 1995 Phys. Rev. Lett. 74, 3724.

Gorski, K. M. ET AL. 1996 Astrophys. J. (Lett.) in press.

Guth, A. H. 1981 Phys. Rev. D 23, 347.

Guth, A. H. \& PI, S.-Y. 1982 Phys. Rev. Lett. 49, 1110.

HamuY, M. ET AL. 1995 Astron. J. 109, 1.

Hawking, S. W. 1982 Phys. Lett. B 115, 295.

Hill, J. E. 1995 Phys. Rev. Lett., 2654.

Hirata, K. S. ET AL. 1992 Phys. Lett. B 280, 146.

Holman, R., Ramond, P. \& Ross, G. G. 1984 Phys. Lett. B 137, 343.

Holtzman, J. A. 1989 Astrophys. J. Suppl. 71, 1.

Hu, W. \& Sugiyama, N. 1995 Phys. Rev. D 51, 2599.

Hu, W. \& White, M. 1996 astro-ph/9602020.

Hu, Y., Turner, M. S. \& Weinberg, E. J. 1994 Phys. Rev. D 49, 3830.

Huchra, J. \& Geller, M. 1989 Science 246, 897.

JaCoby, G. ET AL. 1992 Proc. Astron. Soc. Pac. 104, 599.

Jensen, L. \& Stein-Schabes, J. 1987 Phys. Rev. D 35, 1146.

Jungman, G., Kamionkowski, M., Kosowsky, A. \& Spergel, D. 1996a Phys. Rev. Lett. $\mathbf{7 6}, 1007$.

Jungman, G., Kamionkowski, M., Kosowsky, A. \& Spergel, D. 1996b Phys. Rev. D, in press, astro-ph/9512139.

Kamionkowski, M., Spergel, D. N. \& Sugiyama, N. 1994 Astrophys. J. 426, L57.

Knox, L. 1995 Phys. Rev. D 52, 4307.

Knox, L. \& Turner, M. S. 1993 Phys. Rev. Lett. 70, 371.

Knox, L. \& Turner, M. S. 1994 Phys. Rev. Lett. 73, 3347.

Kochanek, C. S. 1996 Astrophys. J., in press, astro-ph/9510077.

Kofman, L. A., Linde, A. D. \& Einsato, J. 1987 Nature 326, 48. 
Kofman, L. \& Starobinsky, A. A. 1985 Sov. Astron. Lett. 11, 271.

Kolb, E. W. 1991 Physica Scripta T36, 199.

Kolb, E. W. \& Turner, M. S. 1983 Ann. Rev. Nucl. Part. Sci. 33, 645.

Kolb, E. W. \& Turner, M. S. 1990 The Early Universe. Addison-Wesley, Redwood City.

Kosowsky, A., Turner, M. S. \& Watkins, R. 1992 Phys. Rev. Lett. 69, 2026.

Krauss, L. \& Turner, M. S. 1995 Gen. Rel. Grav. 27, 1137.

La, D. \& Steinhardt, P. J. 1991 Phys. Rev. Lett. 62, 376.

Lanzetta, K., Wolfe, A. M. and Turnshek, D. A. 1995 Astrophys. J. 440, 435.

LidDLE, A. ET AL. 1995 astro-ph/9511957.

Liddle, A. \& Lyth, D. 1992 Phys. Lett. B 291, 391.

Liddle, A. \& Lyth, D. 1993 Phys. Repts. 231, 1.

Lidsey, J. E. \& Coles, P. 1992 Mon. Not. Roy. Astron. Soc. 258, 57.

LIDSEY, J. ET AL. 1995 astro-ph/9508078.

Lin, H. ET AL. 1996 Astrophys. J., in press, estro-ph/9602064.

Linde, A. D. 1982 Phys. Lett. B 108, 389.

Linde, A. D. 1983 Phys. Lett. B 129, 177.

Linde, A. D., 1990 Inflation and Quantum Cosmology. Academic Press.

Lucchin, F., Mattarese, S., \& Mollerach, S. 1992 Astrophys. J. 401, L49.

Ma, C. P. \& Bertschinger, E. 1994 Astrophys. J. 434, L5.

Murayama, H. et AL. 1994 Phys. Rev. D 50, R2356.

Novikov, I. D. \& Zel'Dovich, YA. B. 1967 Ann. Rev. Astron. Astrophys. 5, 627.

Novikov, I. D. \& Zel'dovich, YA. B. 1973 Ann. Rev. Astron. Astrophys. 11, 387.

Olive, K. 1990 Phys. Repts. 190, 309.

Ostriker, J. P. 1993 Ann. Rev. Astron. Astrophys. 31, 689.

Ostriker, J. P. \& Steinhardt, P. J. 1995 Nature 377, 600.

PARKe, S. 1995 Phys. Rev. Lett. 74, 839.

Peacock, J. \& Dodds, S. 1994 Mon. Not. Roy. Astron. Soc. 267, 1020.

Peebles, P. J. E. 1984 Astrophys. J. 284, 439.

Peebles, P. J. E., Schramm, D. N., Turner, E., \& Kron, R. 1991 Nature 352, 769.

Peebles P. J. E. \& Yu, J. T. 1970 Astrophys. J. 162, 815.

Perlmutter, S. et Al. 1996 astro-ph/9602122.

Pi, S.-Y. 1984 Phys. Rev. Lett. 52, 1725.

Pogosyan, D. \& Starobinsky, A. A. 1995 astro-ph/9502019.

Preskill, J. 1984 Ann. Rev. Nucl. Part. Sci. 34, 461.

Primack, J. et Al. 1995 Phys. Rev. Lett. 74, 2160.

Randall, L., Soljacic, M. \& Guth, A. H. 1996 hep-ph/9601296.

Ratra, B. 1992 Astrophys. J. 391, L1.

Reiss, A., Kirshner, R. P. \& Press, W. 1995 Astrophys. J. 438, L17.

Rosenberg, L. J. 1995 Particle World 4, 3.

Rubakov, V. A., Sazhin, M. \& Veryaskin, A. 1982 Phys. Lett. B 115, 189.

Rugers, M. \& Hogan, C. J. 1996a Astrophys. J. (Lett.), in press, astro-ph/9512004.

Rugers, M. \& Hogan, C. J. 1996b astro-ph/9603084.

SAlopeK, D. 1992 Phys. Rev. Lett. 69, 3602.

Shafi, Q. \& Stecker, F. 1984 Phys. Rev. Lett. 53, 1292.

Shafi, Q. \& Vilenkin, A. 1984 Phys. Rev. Lett. 52, 691.

Silk, J. \& Turner, M. S. 1986 Phys. Rev. D 35, 419. 
Smoot, G. F. et AL. 1992 Astrophys. J. 396, L1.

Songaila, A. ET AL. 1994 Nature 368, 599.

Souradeep, T. \& Sahni, V. 1992 Mod. Phys. Lett. A 7, 3541.

Starobinsky, A. A. 1982 Phys. Lett. B 117, 175.

Starobinsky, A. A. 1983a Sov. Astron. Lett. 9, 302.

Starobinsky, A. A. 1983b JETP Lett. 37, 66.

Steinhardt, P. J. \& Turner, M. S. 1984 Phys. Rev. D 29, 2162.

Storrie-Lombardi, L. J., McMahon, R. G., Irwin, M. J. \& Hazard, C. 1995 astro$\mathrm{ph} / 9503089$.

Strauss, M. \& Willick, J. 1995 Phys. Repts. 261, 271.

Sugiyama, N. \& Gouda, N. 1992 Prog. Theor. Phys. 88, 803.

Trimble, V. 1987 Ann. Rev. Astron. Astrophys. 25, 425.

Turner, M. S. 1993 Proc. Nat. Acad. Sci. (USA) 90, 4827.

Turner, M. S. 1991 Physica Scripta T36, 167.

Turner, M. S. 1993a Science 262, 861.

Turner, M. S. 1993b Phys. Rev. D 48, 3502.

TURner, M. S. 1996 astro-ph/9607066.

Turner, M. S., Lidsey, J. \& White, M. 1993 Phys. Rev. D 48, 4613.

Turner, M. S., Steigman, G. \& Krauss, L. 1984 Phys. Rev. Lett. 52, 2090.

Turner, M. S. \& Widrow, L. M. 1986 Phys. Rev. Lett. 57, 2237.

Turner, M. S. \& Widrow, L. M. 1988 Phys. Rev. D 37, 2743.

Turner, M. S. \& Wilczek, F. 1990 Phys. Rev. Lett. 65, 3080.

Tytler, D., Fan, K.-M. \& Burles, S. 1996 astro-ph/9603069.

Wampler, E. J. et Al. 1996 Astron. Astrophys., in press, astro-ph/9512084.

Weinberg, S. 1989 Rev. Mod. Phys. 61, 1.

White, D. A. \& Fabian, A. C. 1995 Mon. Not. Roy. Astron. Soc. 273, 72.

White, M. \& Bunn, E. F. 1995 Astrophys. J. (Lett.) 450, 477.

White, M., Scott, D. \& Silk, J. 1995 Science 268, 829.

White, M., Scott, D., Silk, J. \& Davis, M. 1995 Mon. Not. Roy. Astron. Soc. 276, L69.

White, S. D. M. ET AL. 1993 Nature 366, 429.

White, S. D. M., Efstathiou, G. \& Frenk, C. S. 1993 Mon. Not. Roy. Astron. Soc. 262, 1023.

White, S. D. M., Frenk, C. \& Davis, M. 1983 Astrophys. J. 274, L1.

Wilson, M. L. \& Silk, J. 1981 Astrophys. J. 243, 14.

ZeL'Dovich, YA. B. \& Novikov, I. D. 1983 Relativistic Astrophysics (Structure and Evolution of the Universe). Univ. Chicago Press. 